\title{
Retraction Note to: Energy Efficient, Chain Based Clustering Routing Protocol for Wireless Sensor Networks
}

\author{
Subhajit Pal ${ }^{1}$, Debnath Bhattacharyya ${ }^{2}$, and Tai-hoon Kim² \\ ${ }^{1}$ Heritage Institute of Technology \\ Kolkata-700107, India \\ pal.subhajit77@gmail.com \\ ${ }^{2}$ Hannam University \\ Daejeon, Korea \\ debnathesersc.org, taihoonn@empal.com
}

\section{DOI 10.1007/978-3-642-16444-6_83}

The paper starting on page 472 of this volume has been retracted as the vast majority of its contents is included in the paper starting on page 482 of the same volume. 